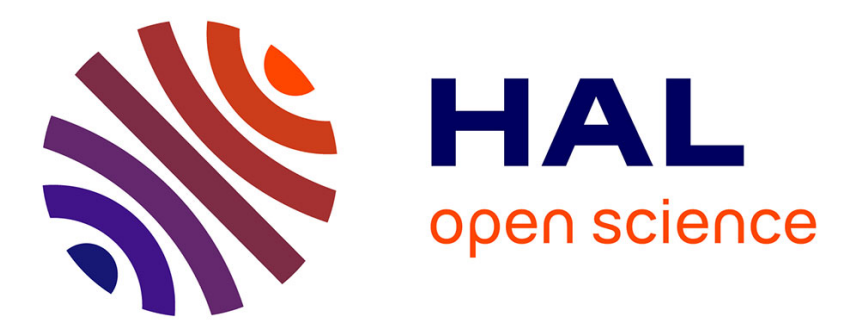

\title{
Contrasted central effects of n-3 versus n- 6 diets on brain functions in diet-induced obesity in minipigs
}

Charles-Henri Malbert, David Val-Laillet, Paul Meurice, Jean-Paul Lalles, Jacques Delarue

\section{To cite this version:}

Charles-Henri Malbert, David Val-Laillet, Paul Meurice, Jean-Paul Lalles, Jacques Delarue. Contrasted central effects of n-3 versus n- 6 diets on brain functions in diet-induced obesity in minipigs. Nutritional Neuroscience, 2022, 25 (7), pp.1453-1465. 10.1080/1028415X.2020.1866881 . hal-03124376

\section{HAL Id: hal-03124376 \\ https://hal.inrae.fr/hal-03124376}

Submitted on 22 Feb 2021

HAL is a multi-disciplinary open access archive for the deposit and dissemination of scientific research documents, whether they are published or not. The documents may come from teaching and research institutions in France or abroad, or from public or private research centers.
L'archive ouverte pluridisciplinaire HAL, est destinée au dépôt et à la diffusion de documents scientifiques de niveau recherche, publiés ou non, émanant des établissements d'enseignement et de recherche français ou étrangers, des laboratoires publics ou privés. 


\title{
Contrasted central effects of $n-3$ versus $n-6$ diets on brain functions in diet-induced obesity in minipigs
}

\author{
Charles-Henri Malbert ${ }^{(1)}$, David Val-Laillet ${ }^{(2)}$, Paul Meurice ${ }^{(2)}$, Jean-Paul
}

Lallès $^{(3)}$, and Jacques Delarue ${ }^{(4)}$

1 - Aniscan Unit, Division of Human Nutrition, INRAE, 35590 Saint-Gilles, France.

2 - INRAE, INSERM, Univ Rennes, Nutrition Metabolisms and Cancer, NuMeCan, 35590 Saint-Gilles, France.

3 - Division of Human Nutrition, INRAE, SDAR, Domaine de la Motte, 35653 Le Rheu, France.

4 - Department of Nutritional Sciences \& Laboratory of Human Nutrition, Hospital University/Faculty of Medicine/University of Brest, France.

Corresponding author's contact information Charles-Henri Malbert

Aniscan Unit, Division of Human nutrition, INRAE

Aniscan, INRAE, 35590 Saint-Gilles, France

Mobile : +33608918989

Email: charles-henri.malbert@inrae.fr 


\begin{abstract}
Introduction: N3 polyunsaturated fatty acids (n-3 PUFAs) exert anti-inflammatory effects for the hypothalamus, but their extra-hypothalamic outcome lack documentation. We evaluated the central consequences of the substitution of saturated fatty acids with n-3 or n-6 PUFA in obesogenic diets.
\end{abstract}

Methods: Twenty-one miniature pigs were fed ad libitum obesogenic diets enriched in fat provided either as lard, fish oil (source for n-3 PUFAs), or sunflower oil (source for n-6 PUFAs) for ten weeks. The blood-brain barrier (BBB) permeability was quantified by CT perfusion. Central autonomic network was evaluated using heart rate variability, and PET 18FDG was performed to assess brain metabolism.

Results: BBB permeability was higher in lard group, but heart rate variability changed only in fish oil group. Brain connectivity analysis and voxel-based comparisons show regional differences between groups except for the cingulate cortex in fish oil vs. sunflower oil groups.

Discussion: The minute changes in brain metabolism in obese pigs feed with fish oil compared with saturated fatty acids were sufficient to induce detrimental changes in heart rate variability. On the contrary, the BBB's decreased permeability in n-3 and n-6 PUFAs groups was protective against an obesity-driven damaged BBB.

Keywords: polyunsaturated fatty acids; brain blood barrier; brain connectivity; brain metabolism; diet induced obesity; miniature pig. 


\section{Introduction}

n-3 PUFAs have beneficial effects on learning and memory, enhance synaptic plasticity and neurogenesis (1), and regulate microglia function (2), (3). They also increase glucose uptake in non-human primates (4), (2). However, these effects were obtained during either n-3 PUFAs supplementation or depletion in an otherwise balanced, non-obesogenic diet (5). Conversely, the central effects of n-3 PUFAs have been poorly studied in the context of obesity and western diets, with the notable exception of the hypothalamus (6). A meta-analysis of 9 randomized clinical trials, concluded to no effect on body mass index in obese (7) but DHA supplementation, a provider of n-3 PUFAs, increased prefrontal cortex activity (8), an area with reduced activity in obese patients (9). Obesity-induced brain inflammation has been partially reversed by dietary supplementation with n-3 PUFAs in mice (10), further supporting the possibility of a possible protective effect of n-3 PUFAs towards the deleterious effects of obesity on the brain (2). Otherwise, obesity causes changes to several cell types belonging to the neurovascular unit that ultimately alter the blood-brain barrier (BBB) integrity (11). However, in HF fed rats (12), these alterations were variable depending on the brain structure ranging from increased permeability in the hippocampus to unchanged permeability in the prefrontal cortex and the striatum. Conversely, increased permeability of cortical structures only (13) was reported in rabbits fed a HF diet enriched in cholesterol. Equally conflicting results were reported for the impact of n-3 PUFAs on the BBB permeability. Hong et al. demonstrated that DHA supplementation in rats $(5 \mathrm{mg} / \mathrm{kg})$ diminished BBB damage induced by middle cerebral artery occlusion (14). Conversely, Yang et al. using the same model of BBB damage established a detrimental effect of DHA (dose of DHA is not reported) with an increase in cerebral ischemia/reperfusion-induced alterations (15). Finally, in another study, n-3 PUFAs were reported to have no effect or contrasted effects depending on the brain structure: positive in the paraventricular nucleus of the hypothalamus, but negative in the anterior pituitary (6).

The present study aimed to assess the cerebral consequences of the long-term substitution of saturated fatty acids (SFAs) with either n-3 PUFAs from fish oil or n-6 PUFAs from sunflower oil within Western-type diets (16). For that purpose, we used the adult Pitman-Moore miniature pig, an animal model known to rapidly develop obesity and insulin resistance in response to a high-fat/high-sucrose diet (17) and to have a relatively large brain with welldefined cerebral circumvolutions (18). We hypothesized that n-3 PUFAs substitution would 
antagonize various facets of brain function as altered by obesity, leading to (i) a regiondependent restoration of the BBB, (ii) an improvement of the reduced prefrontal or limbic glucose metabolism (19), and (iii) a reconditioning of the metabolic network (20). Accordingly, BBB permeability was measured using perfusion CT (21) and analyzed using impulse residue function to extract the quantitative parameters $\mathrm{CBF}$ and $\mathrm{CBV}$ that represent the gold standard to investigate BBB permeability (22). The brain metabolism was obtained region wise using PET ${ }^{18}$ FDG imaging (23) analyzed for regional activations using spatial parameter mapping and metabolic connectivity by network analysis (24). The functional impact of these central changes was investigated with frequency analysis of the heart rate variability, a function of the vagal dorsal complex modulated by higher brain centers (25), and which is a proxy of the autonomic dysfunction in central obesity (26). 


\section{Materials and methods}

The experiment was conducted in accordance with the current ethical standards of the European Community (Directive 2010/63/EU), Agreement No. A35-622 (now C35-275-32). The Regional Ethics Committee in Animal Experimentation of Brittany (France) has validated and approved the entire procedure described in this paper (R-2011-MO-01).

\section{Animals}

A total of 21 female Pitman-Moore miniature pigs, aged 240 days and weighing $23.6 \pm$ $0.60 \mathrm{~kg}$ at the beginning of the experiment were used. The exclusive usage of female animals was dictated by possible gender differences in PUFAs metabolism (27). The animals were ovariectomized before the beginning of the experiment as they were likely to go into one or two heat during the length of the study and because the ovarian cycle induces large variations in brain metabolism (28) and changes in DHA brain concentration (27). The surgery was performed in laparoscopic conditions to limit the experimental bias associated with an open abdomen laparotomy (29). The animals were allowed to recover from surgery during two weeks before the onset of the experiment. The animals were housed individually in $2 \mathrm{~m}^{2}$ cages, enriched with toys to encourage physical exercise. Free visual but limited physical contact between animals was permitted by the design of the cages.

\section{Experimental diets and protocol}

The animals were fed ad libitum once daily at 9:00 a.m. with one of the three diets depending on their assigned experimental group. Refusals were removed daily and weighted. Water was given ad libitum. All the diets supplied had an identical energy density $(18.01 \mathrm{MJ} / \mathrm{kg}$ of feed) (Table 1). They were supplied in about 1.5 times the recommended energy intake of $1.2 \mathrm{MJ} / \mathrm{kg} \mathrm{BW}^{0.75}(30)$ for the duration of the experiment. The experimental diets were designed to generate large differences in n-6 and n-3 PUFAs contents (Table 2) while maintaining about $13 \%$ of crude fat, which is the maximum allowed in pig diet in the context of HF diet without reduction in diet palatability and for making possible the feed granulation.

The animals were divided in three groups ( $\mathrm{n}=7$ each): lard, fish oil (supplying $\mathrm{n}-3$ PUFAs), and sunflower oil (supplying n-6 PUFAs). They were weighed weekly. After 10 weeks of the regimen, CT-scan imaging was performed to evaluate fat deposition and fat-free mass. During 
the same imaging session, contrast medium was also administered in a peripheral vein to challenge the permeability of the BBB that was quantified using CT-based perfusion maps, and heart rate was recorded to evaluate autonomic balance. One week after, brain PET imaging was performed to assess glucose metabolism after the administration of ${ }^{18} \mathrm{FDG}$, a glucose analog radioactive tracer.

\section{HRV measurements}

Heart rate variability (HRV) was analyzed from 5-minute-long electrocardiographic recordings obtained using II derivation (17). Briefly, the electrocardiogram (ECG) was recorded using an ECG machine (GE, USA) in a driven right leg configuration and digitized at $400 \mathrm{~Hz}$ (NI-USB-6008, National instruments, USA) after low pass filtration (analog Butterworth filter set at $200 \mathrm{~Hz}$ ) to cancel aliasing. The digitized signal was streamed continuously as a TDMS (Technical Data Management System) file and analyzed afterward with Labview ${ }^{\circledR}$ biomedical $^{2}$ toolkit (National instruments, USA). After QRS recognition, the RR intervals were detrended and analyzed in the frequency domain with an autoregressive algorithm to extract low (LF 0.04 to $0.15 \mathrm{~Hz}$ ) and high (HF -0.15 to $0.40 \mathrm{~Hz}$ ) frequency components and their ratio. LF (related to sympathetic activity) and HF (related to parasympathetic and notably vagal activity) components were normalized according to the total power of the autoregressive spectrum calculated between 0 to $0.4 \mathrm{~Hz}$.

\section{CT scanning}

CT scanning was performed in isoflurane-anesthetized animals under artificial ventilation (AS3, GE, France). Minimal alveolar concentration (MAC) of isoflurane was set to 1.6 to reduce the possible changes in $\mathrm{CBF}$ due to the anesthetic compound and to cancel the intersubject variations. Ventilation parameters were adapted for a spO2 of $98 \%$ and a $\mathrm{spCO} 2$ of $4.5 \%$.

CT scans for abdominal fat assessment were achieved according to previously validated method (31) using the same CT machine as the one used afterwards for BBB permeability measurement. Scanning was performed using a Hipped Nx/i CT (GE, France) with the animal in ventral recumbency. A pre-heated low-osmolarity nonionic iodine contrast medium (Optiject 300, Guerbet, France) was injected intravenously by a power injector (Angiomat 6000 , Liebel-Flarsheim, USA) as a short bolus of $50 \mathrm{ml}(4 \mathrm{ml} / \mathrm{sec})$ through a catheter in the 
marginal vein of the ear followed by $20 \mathrm{ml}$ of saline solution. First, a non-enhanced axial CT was performed initially to locate the adequate slice for dynamic scan. This slice was centered to the thalamus for later identification of the anterior cerebral artery, which was used for calculation of the arterial input function (32). Second, CT cine mode was performed to dynamically acquire 50 thick slices ( $1 \mathrm{sec}$ per slice, $6 \mathrm{~mm}$ thick, $120 \mathrm{KV}, 200 \mathrm{~mA}$ ). The contrast medium was injected 5 seconds before initiating the dynamic scans.

\section{CT-scan image analysis}

Each brain slice was reconstructed into a 512 x 512 matrix using a smooth kernel filter. Voxel dimensions were anisotropic $0.15 \times 0.15 \times 6 \mathrm{~mm} 3$. CBF, CBV and MTT were calculated semi-automatically using an in-house software written under Labview/IMAQ 2015 IDE (National Instruments, USA). Briefly, perfusion maps were created using a Fourier transformbased deconvolution analysis of the arterial input function with the tissue concentration of contrast medium (33). To quantify perfusion maps, it was assumed that contrast agent concentration was proportional to X-ray attenuation value with a proportionality constant of $1 \mathrm{~g} / \mathrm{mL} / \mathrm{HU}$. Similarly, after automatic segmentation of extra-cerebral tissue, baseline scans were subtracted to perfusion scans assuming a brain density of $1.04 \mathrm{~g} / \mathrm{mL}$. Partial volume effects in the arterial input function were corrected using rescaling of the arterial input function with peak concentration value within the interior sagittal sinus.

The perfusion maps were co-registered to a 3D CT scan template/brain atlas template (34) resliced to the same area and voxel matrix size than the one used for perfusion maps. This allowed obtaining quantitative perfusion parameters for 7 brain areas: frontal, insular, occipital, parietal and temporal cortices plus part of the striatum and the anterior part of the thalamus. Cerebral blood flow (CBF), cerebral blood volume (CBV) and mean transit time (MTT) images were computed and their values averaged for all the 7 brain areas.

\section{PET scanning}

After a 12-h fasting period, the animals were anesthetized using the same conditions than those described for CT-scan measurements. The animals were placed in ventral recumbency on the bed of a whole-body high-resolution PET and a venous catheter was inserted into their left ear in order to inject the radiolabel. The ears and eyes of the animals were sealed with cotton and surgical tape, respectively, to minimize auditory and visual stimulations. The body 
temperature was maintained at $38^{\circ} \mathrm{C}$ by a heating blanket.

The radiolabel $\left({ }^{18} \mathrm{FDG}, 200 \mathrm{MBq}\right.$, IBA France) was injected $45 \mathrm{~min}$ before PET imaging. PET data were acquired on a CTI/Siemens HR+ Scanner in 3D mode (Siemens ECAT, HR+). A 30-min 3D emission scan was performed using an axial field of view of $15.52 \mathrm{~cm}$. It was corrected for attenuation by a 15 -min transmission scan using rotating 68 -Ge rods. Following scatter, dead time and random corrections, PET transaxial images were obtained by iterative reconstruction using a ramp filter (Kernel $\mathrm{FWHM}=6 \mathrm{~mm}$ ) providing 63 contiguous slices. Spatial resolution after reconstruction was $0.64 \mathrm{~mm}$ per pixel in the $\mathrm{x}$ and $\mathrm{y}$ directions and 2.42 mm per pixel in the z-axis. Pixel depth was encoded using the Standard Uptake Value (SUV) method.

\section{PET images analysis}

Images were normalized using normalized mutual information build in PMOD (Pmod Switzerland) with the pig FDG template co-registered with pig brain atlas (34). Due to the anatomical peculiarities of the pig skull, co-registration was performed using data masking. The binary mask was manually drawn for each data set using the masking feature of PMOD. Once co-registered, the images were normalized to the mean SUV of the cerebellum grey matter for each subject (35). We selected the cerebellum grey matter as a reference tissue because its CMRglu, obtained from time-activity curves analysis, was unaffected by obesity in miniature pigs, unlike whole brain CMRglu $(17,20)$. The images were either analyzed pixelwise using statistical brain mapping or VOI-wise (volume of interest) engulfing large brain regions to increase statistical power while minimizing type-2 error. The VOI were selected according to those successfully investigated in obese pigs (19) and obese glucose-intolerant humans (36). Collectively, the cumulative volume of these 7 VOI's accounted for $79 \%$ of the cerebrum grey matter. VOI-based analysis was performed after partial volume correction based on the method for PET brain studies (37). Differences at the voxel level were analyzed using SPM (SPM 12, Wellcome Trust Centre for Neuroimaging, London, UK) with differences between diet groups being investigated using a One-way ( 1 x 3$)$ between-subjects ANOVA model. A significance level of $\mathrm{P} \leq 0.001$ with FDR (False detection rate) correction was used to exclude random brain activation (cluster size $=50$ voxels).

Metabolic connectivity analysis (38) was performed using NetPET software on quantitative 
CMRglu images to identify significant network components (NetPET V1, courtesy of L. Moro, M. Veronese and M. Arcolin). Brain networks were visualized with BrainNet Viewer (39) and the circular graph subroutine (courtesy of P Kassebaum). Brain connectivity matrices were thresholded using the corresponding probability matrices for $\mathrm{P} \leq 0.001$.

\section{Statistical analysis}

Data are presented as means \pm SEM. $\mathrm{P}<0.05$ was considered as significant except for brain image analysis (see below). Data were compared using one and two-way analysis of variance depending on the dataset using Prism 8 (Graphad, USA) unless otherwise notified. Timedependent analyses (changes in body weight) were corrected for multiple comparisons using the Sidak test and T-values were obtained from Tukey pairwise comparison tests performed in Stata 14 (StataCorp, USA). Body weight was analyzed by Ancova with final weight as the dependent variable and pre-diet weight as the covariable. The same procedure was also used for CT-based abdominal fat and lean mass measurements. 


\section{Results}

\section{Body composition}

The body weight of the pigs steadily increased during the 12 weeks of the dietary intervention from $23.6 \pm 0.60 \mathrm{~kg}$ to $41.7 \pm 0.82 \mathrm{~kg}$. This $43.4 \%$ increase was not significantly different between groups. A slight but significant difference between PUFA's groups and SFA's group was observed at 63 and 70-days after the beginning of feeding the experimental diets ( $\mathrm{P}=0.0419$ and 0.0161 , respectively) but it was not observed anymore 77 and 84 days post intervention $(\mathrm{P}=0.3930$ and 0.0508 , respectively). After 12 weeks of dietary intervention, visceral fat mass, and lean mass (representing the cumulative volumes of the gluteus medius, longissimus dorsi, iliocostalis, psoas and serratus ventralis muscles) evaluated by CT-scans were similar between the 3 groups (Figure 1).

\section{Heart rate variability}

After dietary intervention, heart rate was not different between groups $(92 \pm 5.6,88 \pm 5.7$ and $99 \pm 4.0$ beat per minute for lard, fish oil and sunflower oil respectively, $\mathrm{P}=0.327$ ). However, the LF component of the variability spectrum was significantly higher for the fish oil group (Figure 2, $\mathrm{P}=0.048$ ) while it was not significantly different between the lard and sunflower oil groups $(\mathrm{P}=0.971)$. There was no difference for the HF component $(\mathrm{P}=0.083)$. Therefore, the LF/HF ratio reflected a sympathetic LF component significantly higher for the fish oil group $(\mathrm{P}=0.043)$.

\section{Blood-brain barrier (BBB) permeability}

Cerebral blood flow (CBF), i.e. the instantaneous capillary flow in the brain parenchyma as a whole, was significantly modified by the diet $(\mathrm{P}<0.001)$ but this was not region-specific $(\mathrm{P}>0.999)$. The same feature was also observed for CBV for which the modifications were also not region-specific. Mean CBF and CBV measured for all brain areas were about doubled for lard group as compared to fish oil and sunflower oil groups (Figure 3). There were no differences between fish oil and sunflower groups for CBF and CBV ( $\mathrm{P}=0.535$ and 0.610 respectively). Mean transit time, which measures the length of time a certain volume of blood spends in the cerebral capillary circulation, was not different between groups $(\mathrm{P}>0.999)$ or between brain areas $(\mathrm{P}>0.999)$. 


\section{Glucose brain metabolism}

Ancova analysis of voxel-wise linear comparisons between the three diet groups did not show any difference at $\mathrm{P}<0.001 \mathrm{FDR}$ corrected. Using a smaller yet physically realistic cluster size (cluster size $=30$ instead of 50 voxels) or removing grand-mean correction were equally ineffective to identify activated or de-activated clusters. T-test comparisons were also unable to point out significant brain activation with the notable exception of fish oil group that exhibited a significant activation of the dorsal right cingulate cortex $(\mathrm{p}=0.001)$ in comparison to the sunflower group (Figure 4).

Volume of interest (VOI) analysis, a more powerful statistical analysis at the expense of spatial localization, was also unable to identify a statistical difference between glucose metabolism irrespective of the area under scrutiny. Nevertheless, the glucose uptake was significantly greater for the fish oil group compared to sunflower oil group for the cerebrum, i.e. the encephalon without the cerebellum (Figure 4). This suggests that the metabolic alterations, if any, were more widely distributed in the brain.

Metabolic connectivity analysis showed that all networks were thalamic-centric irrespective of the group. However, significant changes in networks were observed between groups (Figure 5). For the lard group, the network engulfed limbic structures encompassing the putamen and the insula. For the fish oil group, projections of the network were observed in the right cingulate cortex whereas the network remained thalamic-centric for the sunflower oil group. 


\section{Discussion}

\section{$\underline{\text { Summary of main findings }}$}

This is the first study that aimed at quantifying comprehensively the effects of large amounts of dietary PUFAs fed chronically on brain function in an obesogenic diet context. We demonstrated that a diet rich in n-3 PUFAs was associated with a significant change of the heart rate variability (HRV), indicating a parallel modification of the autonomic tone. The $n-3$ and $n-6$ PUFA-containing diets were equally effective to restrict the BBB permeability compared to the SFAs-rich diet. Despite these large changes in BBB permeability, the brain glucose metabolism was almost not regionally modified unlike the metabolic networks that presented extensive variations.

\section{Changes in heart rate variability}

While primarily controlled by the dorsal vagal complex, substantial evidence showed that HRV correlates with the central autonomic network, particularly the cingulate cortex, the insula, and the putamen $(40,41)$. Unsurprisingly, the same structures were found to be part of the network instantiated in fish oil group while LF/HF was also primarily modified in the same group. Indeed, we found that a n-3 PUFAs-rich diet was associated with a resting network extending to the cingulate cortex, a structure that was also found activated when compared with sunflower oil group. Altogether, these data demonstrate that the replacement, within a high-fat diet, of SFAs by a large amount of n-3 PUFAs was able to modify brain metabolism and function while in a resting and unstimulated condition during brain imaging. Such unconsidered feature so far (42) makes crucial the nature of the diet-related HRV change: an increase in LF spectral component. The engagement of LF component, presumed to represent the sympathetic activity, in the n-3 PUFAs obese animals is opposite to the increase in HF observed by Ninio et al (43) in DHA/EPA supplemented obese patients both at rest and during sub-maximal exercise. This is also at odds with our previous observations in humans, when we showed that n-3 PUFAs from fish oil decreased sympatho-adrenal activity in healthy humans submitted to a mental stress on one hand (44), and in haemodialysis patients on the other (45). The n-3 PUFAs-induced increase in LF in our obese minipigs could be detrimental if extrapolable to obese patients with a risk for coronary artery disease (46). However, the usefulness of $\mathrm{LF} / \mathrm{HF}$ ratio as a predictor for heart disease in the context of obesogenic diet relies on incomplete epidemiological evidence (26). 


\section{Alterations of the brain blood barrier}

In small animals, brain blood barrier integrity is classically inferred from brain histology after a carotid infusion of Evans blue, a simple solution but with severe limitations, especially for the BBB permeability quantification (47). We used perfusion computed tomography associated with compartmental modeling as a more accurate proxy since it is sensitive enough to detect permeability default in inflammatory diseases without the limitations of dye injection (48). The analysis of the fate of the tracer within the brain enable us to extract three parameters which are equally essential to characterize the permeability of the BBB: CBF represents the instantaneous capillary flow in the brain, CBV describes the blood volume of the cerebral capillaries and venules per cerebral tissue volume and MTT measures the length of time a particular volume of blood spend in the cerebral capillary circulation. The larger CBF in the lard group compared to the low-SFAs groups was expected since it reflected BBB integrity that is challenged, particularly at the cortical level in some animal models of obesity (49). The paralleled CBV increase in the lard group was novel information since we were unaware of such measurement in obese animals or patients. Taken together with the increase in CBF, CBV changes were a quantitative objective translation of brain hyper-perfusion (50). The increased flow was somewhat unexpected since, in obese type-2 diabetes patients, several studies have reported a decrease in CBF (51). However, these reports were based on SPECT and ASL imaging that concentrate more on the transfer coefficients of water than on microcrystalline solutions such as the one used for CT-based CBF measurements (52). Nevertheless, both fish oil and sunflower oil diets were able to reduce significantly CBF and CBV compared to the standard high-fat western diet, confirming the protective role of unsaturated fat on the BBB permeability (53). Unfortunately, it is not possible to correlate these changes with the underlying cellular and metabolic processes responsible for the BBB permeability, i.e., the interplay between the intracellular and trans-membrane tight junctions, the enzymatic-driven metabolism of blood-borne products, and the numerous transport systems (21).

\section{Modifications of brain metabolism and networks}

The small changes in brain metabolism induced by the otherwise significant differences in the origin of fat in the experimental diets were not expected. To ascertain the robustness of the otherwise small deviations in glucose metabolism between groups, we tested several statistical strategies. They included VOI-based measurement that were thought less prone to type-II error 
and were statistically more powerful at the expense of spatial accuracy than spatial parameter mapping. The converging results from these multiple analytic sources argue for the lack of localized alteration of brain metabolism. On the contrary, we found substantial changes between groups in the metabolic connectivity, which spread towards the insula and the putamen for lard group compared to the low-SFAs groups. Similar changes have been already described in obese animals (20) and patients (54) supporting the role of an altered communication with the reward structures that, in turn, account for the obesity-related alterations in the reward processing of food stimuli (55). Cingulate cortex expansion of the otherwise thalamic-centric network, present in all groups was identified in n-3 group. Altered connectivity was observed also in obese patients between the anterior cingulate cortex and the thalamus using resting state functional magnetic resonance imaging (fMRI) (56). Since fMRI was able to identify the direction of the change, it was possible to confirm that obesity reduced the activity of the cingulate cortex. On the contrary, in healthy older adults, dietary fish oil supplementation increases BOLD signal in the posterior cingulate cortex during higher working memory load, suggesting enhanced neuronal response to working memory challenge (57). A reverse relationship was found in healthy adults with DHA supplementation resulting in a negative correlation between the amount of circulating DHA and the glucose uptake by the anterior cingulate (58). Unfortunately, our analysis of brain network is semi-quantitative compared to what could have been performed using dynamic PET or fMRI (59). We acknowledge that the changes in brain glucose metabolism might be underestimated because we used static PET image recorded 45 minutes after the radiolabeled compound injection instead of a dynamic PET sequence (60). Nevertheless, in grey mouse lemur, a species for which a tridimensional brain atlas is missing hence limiting interpretation, $n-3$ PUFAs supplementation increased the brain glucose metabolism in a non-regional specific manner (61).

\section{Limitations of the study}

The amount of PUFAs contained in the experimental diets is arguably large compared to the dose used in dietary interventions in patients with neurodegenerative diseases or mood disorders (2). Nevertheless, the EFSA Panel on Dietetic Products, Nutrition and Allergies stated that «the available data are not sufficient to establish a tolerable upper intake level for n-3 PUFA (DHA, EPA, and DPA, individually or combined) for any population group» (62). Based on mean daily food intake of $400 \mathrm{~g}$, the amount of EPA supplied to pigs in fish oil group was in the same range that reported in high EU consumers of fatty fish $(3,5$ versus $2,7 \mathrm{~g}$ per 
day). Furthermore, the dose of DHA consumed by the fish oil group is also close that found without toxicity concerns ( $6 \mathrm{~g}$ per day) in humans (63).

\section{$\underline{\text { Implications for future research and clinical practice }}$}

In conclusion, the permeability of the BBB in n-3 and n-6 PUFAs groups was about halved compared to the saturated fat group. This protective pattern against an obesity-driven damaged BBB was not region-specific and was probably at the origin of the insubstantial regional changes of brain metabolism. Despite the absence of focal brain activation in n-3 PUFAs group characteristics of the obese brain, the extension of the resting network towards the cingulate cortex could explain an alteration of heart rate variability with an increase in LF spectral component. The large amount of PUFA present in n-3 diet, while protecting against the damage of the BBB, could be detrimental for heart function. The importance of the heart rhythm dysfunction in the context of long-term substitution of saturated fatty acids with n-3 PUFAs in Western-type diets warrants further investigations on heart perfusion and metabolism. 


\section{Acknowledgement and statements}

Acknowledgement: The authors thank staff of the UE3P unit for animal care, Alain Chauvin, Mickael Genissel, Julien Georges, Francis Le Gouevec, and Vincent Piedvache. We also thank Jean-François Rouault for the production of the experimental diets.

Disclosure Statement and competing interests: The authors have no conflicts of interest to declare.

Guarantor's statement: Charles-Henri Malbert is the guarantor of this work and, as such, had full access to all the data in the study and takes responsibility for the integrity of the data and the accuracy of the data analysis.

Funding sources - The study was conducted with the support of the Brittany council which we thank.

Authors' contribution - C-H.M. planned the experiments, conducted the studies, analyzed the data, and was primarily responsible for writing the manuscript. D.V-L. conducted the studies and contributed to writing the manuscript. P.M. contributed to the analysis of the data. J-P.L planned the experiments, formulated the experimental diet and contributed to writing the manuscript. J.D. contributed to writing the manuscript. 


\section{Bibliography}

1. Dyall SC: Long-chain omega-3 fatty acids and the brain: a review of the independent and shared effects of EPA, DPA and DHA. Front Aging Neurosci 7:52, 2015

2. Laye S, Nadjar A, Joffre C, Bazinet RP: Anti-Inflammatory Effects of Omega-3 Fatty Acids in the Brain: Physiological Mechanisms and Relevance to Pharmacology. Pharmacological Reviews 70:12-38, 2018

3. Desale SE, Chinnathambi S: Role of dietary fatty acids in microglial polarization in Alzheimer's disease. J Neuroinflammation 17:93, 2020

4. Pifferi F, Dorieux O, Castellano CA, Croteau E, Masson M, Guillermier M, Van Camp N, Guesnet P, Alessandri JM, Cunnane S, Dhenain M, Aujard F: Long-chain n-3 PUFAs from fish oil enhance resting state brain glucose utilization and reduce anxiety in an adult nonhuman primate, the grey mouse lemur. J Lipid Res 56:1511-1518, 2015

5. Larrieu T, Laye S: Food for Mood: Relevance of Nutritional Omega-3 Fatty Acids for Depression and Anxiety. Frontiers in Physiology 92018

6. Kuo YT, So PW, Parkinson JR, Yu WS, Hankir M, Herlihy AH, Goldstone AP, Frost GS, Wasserfall C, Bell JD: The combined effects on neuronal activation and blood-brain barrier permeability of time and n-3 polyunsaturated fatty acids in mice, as measured in vivo using MEMRI. Neuroimage 50:1384-1391, 2010

7. Zhang YY, Liu W, Zhao TY, Tian HM: Efficacy of omega-3 polyunsaturated fatty acids supplementation in managing overweight and obesity: A meta-analysis of randomized clinical trials. The journal of nutrition 1-6, 2017

8. McNamara RK, Able J, Jandacek R, Rider T, Tso P, Eliassen JC, Alfieri D, Weber W, Jarvis K, DelBello MP, Strakowski SM, Adler CM: Docosahexaenoic acid supplementation increases prefrontal cortex activation during sustained attention in healthy boys: a placebo-controlled, dose-ranging, functional magnetic resonance imaging study. The American Journal of Clinical Nutrition 91:1060-1067, 2010

9. Volkow ND, Wang GJ, Telang F, Fowler JS, Goldstein RZ, Alia-Klein N, Logan J, Wong C, Thanos PK, Ma Y, Pradhan K: Inverse association between BMI and prefrontal metabolic activity in healthy adults. Obesity (Silver Spring) 17:60-65, 2009

10. de Mello AH, Schraiber RDB, Goldim MPDS, Garcez ML, Gomes ML, de Bem Silveira G, Zaccaron RP, Schuck PF, Budni J, Silveira PCL, Petronilho F, Rezin GT: Omega-3 Fatty Acids Attenuate Brain Alterations in High-Fat Diet-Induced Obesity Model. Molecular Neurobiology 56:513-524, 2019

11. Rhea EM, Salameh TS, Logsdon AF, Hanson AJ, Erickson MA, Banks WA: Blood-Brain Barriers in Obesity. AAPS J 19:921-930, 2017

12. Davidson TL, Monnot A, Neal AU, Martin AA, Horton JJ, Zheng W: The effects of a high-energy diet on hippocampal-dependent discrimination performance and blood-brain barrier integrity differ for diet-induced obese and diet-resistant rats. Physiol Behav 107:26-33, 2012

13. Ghribi O, Golovko MY, Larsen B, Schrag M, Murphy EJ: Deposition of iron and Bamyloid plaques is associated with cortical cellular damage in rabbits fed with long-term cholesterol-enriched diets. Journal of Neurochemistry 99:438-449, 2006

14. Hong SH, Khoutorova L, Bazan NG, Belayev L: Docosahexaenoic acid improves behavior and attenuates blood-brain barrier injury induced by focal cerebral ischemia in rats. Exp Transl Stroke Med 7:3, 2015

15. Yang DY, Pan HC, Yen YJ, Wang CC, Chuang YH, Chen SY, Lin SY, Liao SL, Raung SL, Wu CW, Chou MC, Chiang AN, Chen CJ: Detrimental effects of post-treatment with fatty acids on brain injury in ischemic rats. Neurotoxicology 28:1220-1229, 2007 
16. Kopp W: How Western Diet And Lifestyle Drive The Pandemic Of Obesity And Civilization Diseases. Diabetes, Metabolic Syndrome and Obesity: Targets and Therapy Volume 12:2221-2236, 2019

17. Malbert C-H, Picq C, Divoux J-L, Henry C, Horowitz M: Obesity-associated alterations in glucose metabolism are reversed by chronic bilateral stimulation of the abdominal vagus nerve. Diabetes 66:848-857, 2017

18. Sauleau P, Lapouble E, Val-Laillet D, Malbert CH: The pig model in brain imaging and neurosurgery. Animal 3:1138-1151, 2009

19. Val-Laillet D, Layec S, Guérin S, Meurice P, Malbert CH: Changes in brain activity after a diet-induced obesity. Obesity (Silver Spring) 19:749-756, 2011

20. Bahri S, Horowitz $\mathrm{M}$, Malbert $\mathrm{CH}$ : Inward glucose transfer accounts for insulindependent increase in brain glucose metabolism associated with diet-induced obesity. Obesity (Silver Spring) 2018

21. Avsenik J, Bisdas S, Popovic KS: Blood-brain barrier permeability imaging using perfusion computed tomography. Radiology and Oncology 49:107-114, 2015

22. Hoeffner EG, Case I, Jain R, Gujar SK, Shah GV, Deveikis JP, Carlos RC, Thompson BG, Harrigan MR, Mukherji SK: Cerebral perfusion CT: technique and clinical applications. Radiology 231:632-644, 2004

23. Biraben A, Guerin S, Bobillier E, Malbert $\mathrm{CH}$ : Central activation after chronic vagus nerve stimulation in pigs: contribution of functional imaging. Bulletin Academie Veterinaire France 1612008

24. Malbert C-H, Horowitz M, Young RL: Low-calorie sweeteners augment tissue-specific insulin sensitivity in a large animal model of obesity. European journal of nuclear medicine and molecular imaging 1-12, 2019

25. Jeanne M, Logier R, De Jonckheere J, Tavernier B: Heart rate variability during total intravenous anesthesia: effects of nociception and analgesia. Auton Neurosci 147:91-96, 2009

26. Damodaran A, Kabali B: Autonomic dysfunction in central obesity. World Journal of Medical Sciences 8:118-122, 2013

27. Lin Y-H, Brown JA, DiMartino C, Dahms I, Salem Jr. N, Hibbeln JR: Differences in long chain polyunsaturates composition and metabolism in male and female rats. Prostaglandins, Leukotrienes and Essential Fatty Acids 113:19-27, 2016

28. $\mathrm{Hu}$ Y, Xu Q, Li K, Zhu H, Qi R, Zhang Z, Lu G: Gender differences of brain glucose metabolic networks revealed by FDG-PET: evidence from a large cohort of 400 young adults. PLoS One 8:e83821, 2013

29. Miranda A, Peles S, Rudolph C, Shaker R, Sengupta JN: Altered visceral sensation in response to somatic pain in the rat访. Gastroenterology 126:1082-1089, 2004

30. Bollen PJA, Hansen AK, Alstrup AKO: The Laboratory Swine, Second Edition. 124, 2010

31. Val-Laillet D, Blat S, Louveau I, Malbert CH: A computed tomography scan application to evaluate adiposity in a minipig model of human obesity. Br J Nutr 104:1719-1728, 2010

32. Mori S, Obata T, Nakajima N, Ichihara N, Endo M: Volumetric perfusion CT using prototype 256-detector row CT scanner: preliminary study with healthy porcine model. AJNR American journal of neuroradiology 26:2536-2541, 2005

33. Fieselmann A, Kowarschik M, Ganguly A, Hornegger J, Fahrig R: Deconvolution-Based CT and MR Brain Perfusion Measurement: Theoretical Model Revisited and Practical Implementation Details. International Journal of Biomedical Imaging 2011:1-20, 2011 
34. Saikali S, Meurice P, Sauleau P, Eliat PA, Bellaud P, Randuineau G, Vérin M, Malbert $\mathrm{CH}$ : A three-dimensional digital segmented and deformable brain atlas of the domestic pig. J Neurosci Methods 192:102-109, 2010

35. Dukart J, Mueller K, Horstmann A, Vogt B, Frisch S, Barthel H, Becker G, Möller HE, Villringer A, Sabri O, Schroeter ML: Differential effects of global and cerebellar normalization on detection and differentiation of dementia in FDG-PET studies. Neuroimage 49:1490-1495, 2010

36. Hirvonen J, Virtanen KA, Nummenmaa L, Hannukainen JC, Honka MJ, Bucci M, Nesterov SV, Parkkola R, Rinne J, Iozzo P, Nuutila P: Effects of insulin on brain glucose metabolism in impaired glucose tolerance. Diabetes 60:443-447, 2011

37. Rousset OG, Collins DL, Rahmim A, Wong DF: Design and Implementation of an Automated Partial Volume Correction in PET: Application to Dopamine Receptor Quantification in the Normal Human Striatum. Journal of Nuclear Medicine 49:10971106, 2008

38. Yakushev I, Drzezga A, Habeck C: Metabolic connectivity. Current Opinion in Neurology 30:677-685, 2017

39. Xia M, Wang J, He Y: BrainNet Viewer: a network visualization tool for human brain connectomics. PLoS One 8:e68910, 2013

40. Chang C, Metzger CD, Glover GH, Duyn JH, Heinze H-J, Walter M: Association between heart rate variability and fluctuations in resting-state functional connectivity. NeuroImage 68:93-104, 2013

41. Wei L, Chen $\mathrm{H}, \mathrm{Wu}$ GR: Heart rate variability associated with grey matter volumes in striatal and limbic structures of the central autonomic network. Brain Res 1681:14-20, 2018

42. Christensen JH: Omega-3 polyunsaturated Fatty acids and heart rate variability. Front Physiol 2:84, 2011

43. Ninio DM, Hill AM, Howe PR, Buckley JD, Saint DA: Docosahexaenoic acid-rich fish oil improves heart rate variability and heart rate responses to exercise in overweight adults. British Journal of Nutrition 100:1097-1103, 2008

44. Delarue J, Matzinger O, Binnert C, Schneiter P, Chiolero R, Tappy L: Fish oil prevents the adrenal activation elicited by mental stress in healthy men. Diabetes \& metabolism 29:289-295, 2003

45. Delarue J, Guillodo M-P, Guillerm S, Elbaz A, Marty Y, Clèdes J: Fish oil attenuates adrenergic overactivity without altering glucose metabolism during an oral glucose load in haemodialysis patients. British Journal of Nutrition 99:1041-1047, 2008

46. TFOTESOCTNASOPE: Heart Rate Variability. Circulation 93:1043-1065, 1996

47. Saunders NR, Dziegielewska KM, Møllgård K, Habgood MD: Markers for blood-brain barrier integrity: how appropriate is Evans blue in the twenty-first century and what are the alternatives. Front Neurosci 9:385, 2015

48. Dankbaar JW, Hom J, Schneider T, Cheng S-C, Lau BC, van der Schaaf I, Virmani S, Pohlman S, Dillon WP, Wintermark M: Dynamic perfusion CT assessment of the bloodbrain barrier permeability: first pass versus delayed acquisition. AJNR American journal of neuroradiology 29:1671-1676, 2008

49. Davidson TL, Kanoski SE, Schier LA, Clegg DJ, Benoit SC: A potential role for the hippocampus in energy intake and body weight regulation. Curr Opin Pharmacol 7:613616,2007

50. Sourbron S, Ingrisch M, Siefert A, Reiser M, Herrmann K: Quantification of cerebral blood flow, cerebral blood volume, and blood-brain-barrier leakage with DCE-MRI. Magn Reson Med 62:205-217, 2009 
51. van Golen LW, Huisman MC, Ijzerman RG, Hoetjes NJ, Schwarte LA, Lammertsma AA, Diamant M: Cerebral blood flow and glucose metabolism measured with positron emission tomography are decreased in human type 1 diabetes. Diabetes 62:2898-2904, 2013

52. Koh TS, Tan CKM, Cheong LHD, Lim CCT: Cerebral perfusion mapping using a robust and efficient method for deconvolution analysis of dynamic contrast-enhanced images. 32:643-653, 2006

53. de Mello AH, Uberti MF, de Farias BX, de Souza NAR, Rezin GT: n-3 PUFA and obesity: from peripheral tissues to the central nervous system. Br J Nutr 119:1312-1323, 2018

54. Marques-Iturria I, Scholtens LH, Garolera M, Pueyo R, García-García I, GonzálezTartiere P, Segura B, Junqué C, Sender-Palacios MJ, Vernet-Vernet M, Sánchez-Garre C, de Reus MA, Jurado MA, van den Heuvel MP: Affected connectivity organization of the reward system structure in obesity. Neuroimage 111:100-106, 2015

55. Volkow ND, Wang GJ, Baler RD: Reward, dopamine and the control of food intake: implications for obesity. Trends Cogn Sci 15:37-46, 2011

56. Wijngaarden MA, Veer IM, Rombouts SA, van Buchem MA, Willems van Dijk K, Pij1 $\mathrm{H}$, van der Grond $\mathrm{J}$ : Obesity is marked by distinct functional connectivity in brain networks involved in food reward and salience. Behav Brain Res 287:127-134, 2015

57. Boespflug EL, McNamara RK, Eliassen... JC: Fish oil supplementation increases eventrelated posterior cingulate activation in older adults with subjective memory impairment. The journal of nutrition 2016

58. Sublette ME, Milak MS, Hibbeln JR, Freed PJ, Oquendo MA, Malone KM, Parsey RV, Mann JJ: Plasma polyunsaturated fatty acids and regional cerebral glucose metabolism in major depression. Prostaglandins Leukot Essent Fatty Acids 80:57-64, 2009

59. Di X, Biswal BB, Alzheimer's DNI: Metabolic brain covariant networks as revealed by FDG-PET with reference to resting-state fMRI networks. Brain Connect 2:275-283, 2012

60. Lammertsma AA: Forward to the Past: The Case for Quantitative PET Imaging. $J$ Nucl Med 58:1019-1024, 2017

61. Pifferi F, Dorieux O, Castellano C-A, Croteau E, Masson M, Guillermier M, Van Camp N, Guesnet P, Alessandri J-M, Cunnane S, Dhenain M, Aujard F: Long-chain n-3 PUFAs from fish oil enhance resting state brain glucose utilization and reduce anxiety in an adult nonhuman primate, the grey mouse lemur. Journal of Lipid Research 56:1511-1518, 2015

62. EFSA Panel on Dietetic Products NAANDA: Scientific Opinion on the Tolerable Upper Intake Level of eicosapentaenoic acid (EPA), docosahexaenoic acid (DHA) and docosapentaenoic acid (DPA). EFSA Journal 102012

63. Lien EL: Toxicology and safety of DHA. Prostaglandins, Leukotrienes and Essential Fatty Acids 81:125-132, 2009 
Table 1: Composition and nutritional value of the experimental diets

\begin{tabular}{|l|c|c|c|}
\hline \multicolumn{1}{|c|}{ Composition (\%) } \\
\hline Wheat & Lard & Fish oil & Sunflower oil \\
\hline Soybean meal & 45 & 45 & 45 \\
\hline Lard & 17 & 17 & 17 \\
\hline Fish oil & 12 & - & - \\
\hline Sunflower oil & - & 12 & 12 \\
\hline Corn starch & - & - & 11 \\
\hline Beet pulp & 11 & 11 & 5.1 \\
\hline Cane molasses & 5.1 & 5.1 & 5 \\
\hline Beet molasses & 5 & 5 & 2 \\
\hline Calcium carbonate & 2 & 2 & 1.7 \\
\hline Oligoelements and vitamins & 1.7 & 1.7 & 0.5 \\
\hline Sodium chloride & 0.5 & 0.5 & 0.3 \\
\hline Dicalcium phosphate & 0.3 & 0.3 & 0.4 \\
\hline
\end{tabular}

Nutritional value (calculated; \% or $\mathrm{MJ} / \mathrm{kg}$ )

\begin{tabular}{|l|c|c|c|}
\hline Metabolisable energy & 18.01 & 18.01 & 18.01 \\
\hline Net energy & 11.79 & 11.79 & 11.79 \\
\hline Dry matter & 88.3 & 88.3 & 88.3 \\
\hline Crude protein & 13.37 & 13.37 & 13.37 \\
\hline Crude fat & 13.13 & 13.13 & 13.13 \\
\hline Cellulose & 2.92 & 2.92 & 2.92 \\
\hline Minerals & 5.67 & 5.67 & 5.67 \\
\hline
\end{tabular}


Table 2 - Measured fatty acids (FA) composition of the three experimental diets

\begin{tabular}{|c|c|c|c|}
\hline FA $\%$ of total FA & Lard & Fish oil & Sunflower oil \\
\hline Crude fat & 12.7 & 11.9 & 12.3 \\
\hline Saturated FA & 35.4 & 28.1 & 11.7 \\
\hline $\mathrm{C} 18: 0$ & 9.9 & 4.6 & 3.69 \\
\hline $\mathrm{C} 16: 0$ & 23.9 & 18.5 & 7.35 \\
\hline C18:1 (OA) & 42.0 & 14.5 & 26.1 \\
\hline PUFA & 16.5 & 47.2 & 60.3 \\
\hline C18:2 n-6c (LA) & 14.4 & 8 & 59.7 \\
\hline C18:3 n-3 (ALA) & 1.29 & 1.06 & 0.56 \\
\hline $\mathrm{C} 18: 4 \mathrm{n}-3$ & 0.04 & 1.11 & 0 \\
\hline $\mathrm{C} 20: 5$ n-3 (EPA) & 0 & 7.5 & 0 \\
\hline C22:6 n-3 (DHA) & 0 & 23.8 & 0 \\
\hline Total n-6 & 14.7 & 10.2 & 59.8 \\
\hline Total n-3 & 1.6 & 36.8 & 0.6 \\
\hline$n-6 / n-3$ & 8.9 & 0.3 & 107.4 \\
\hline
\end{tabular}


Figure 1: A - Changes in pigs' live body weight in the three experimental groups: Lard, Fish oil and Sunflower oil. * different at $\mathrm{P}<0.05$ between Lard and Fish oil. $\$$ different at $\mathrm{P}<0.05$ between Lard and Sunflower oil. B - Body composition obtained from image analysis of the CT-scan slices located at T13, L2 and L3 vertebra. Note that the amount of fat (including subcutaneous and abdominal fat), abdominal fat and lean tissue mass were not significantly different between groups.

Figure 2: Spectral analysis of the heart rate variability (HRV) obtained from 5-minute-long recordings. High-frequency component, representative of the parasympathetic (vagal) cardiac modulation, was unchanged irrespective of the diet whereas Fish oil group presented a significant increase in the low frequency sympathetic component and consequently higher $\mathrm{LF} / \mathrm{HF}$ ratio.

Figure 3: A - Cerebral flow (CBF) coded images obtained from the analysis of the injected dynamic CT image. The insert represents the seven brain structures used for the calculation of rCBF (see text). B - CBF, CBV and MTT calculated as a mean over the 7 regions of interest. * Significantly different $(\mathrm{P}<0.05)$ from Lard and Sunflower oil, $\neq$ significantly different $(\mathrm{P}$ $<0.05)$ from Lard group.

Figure 4: A - Statistical parameter mapping image of the differences in normalized glucose uptake relative to the cerebellum between fish oil and sunflower oil groups (in yellow) overlaid on T1 coded atlas. Note the activation of the dorsal right cingulate cortex. B - Tridimensional representation of the dorsal cingulate cortex over a porcine brain glass. $\mathrm{C}$ - Three-dimensional brain representation showing the 7 volumes of interest (VOI). D - VOI analysis of the regional glucose uptake. Cerebrum values were cumulative uptake divided by the number of voxels. $\$$ Significantly different from Lard group $(\mathrm{P}<0.05) . *$ Significantly different from Lard and Fish oil group $(\mathrm{P}<0.05)$.

Figure 5: Metabolic connectivity analysis for Lard, Fish oil and Sunflower oil groups. Left - Metabolic connectivity matrices were calculated using NetPET software. Components were presented using BrainNet Viewer with a threshold of $\mathrm{P} \leq 0.005$ on the probability map associated to the correlation map. The unconnected nodes were not represented to ease visual comparison. The thickness of the edges symbolized the intensity of the correlation. Right Metabolic connectivity represented as circular graphs with a threshold of $\mathrm{P} \leq 0.001$. The description of the nodes and their abbreviations were given in supplementary information 1 . 
A

\section{Body weight}

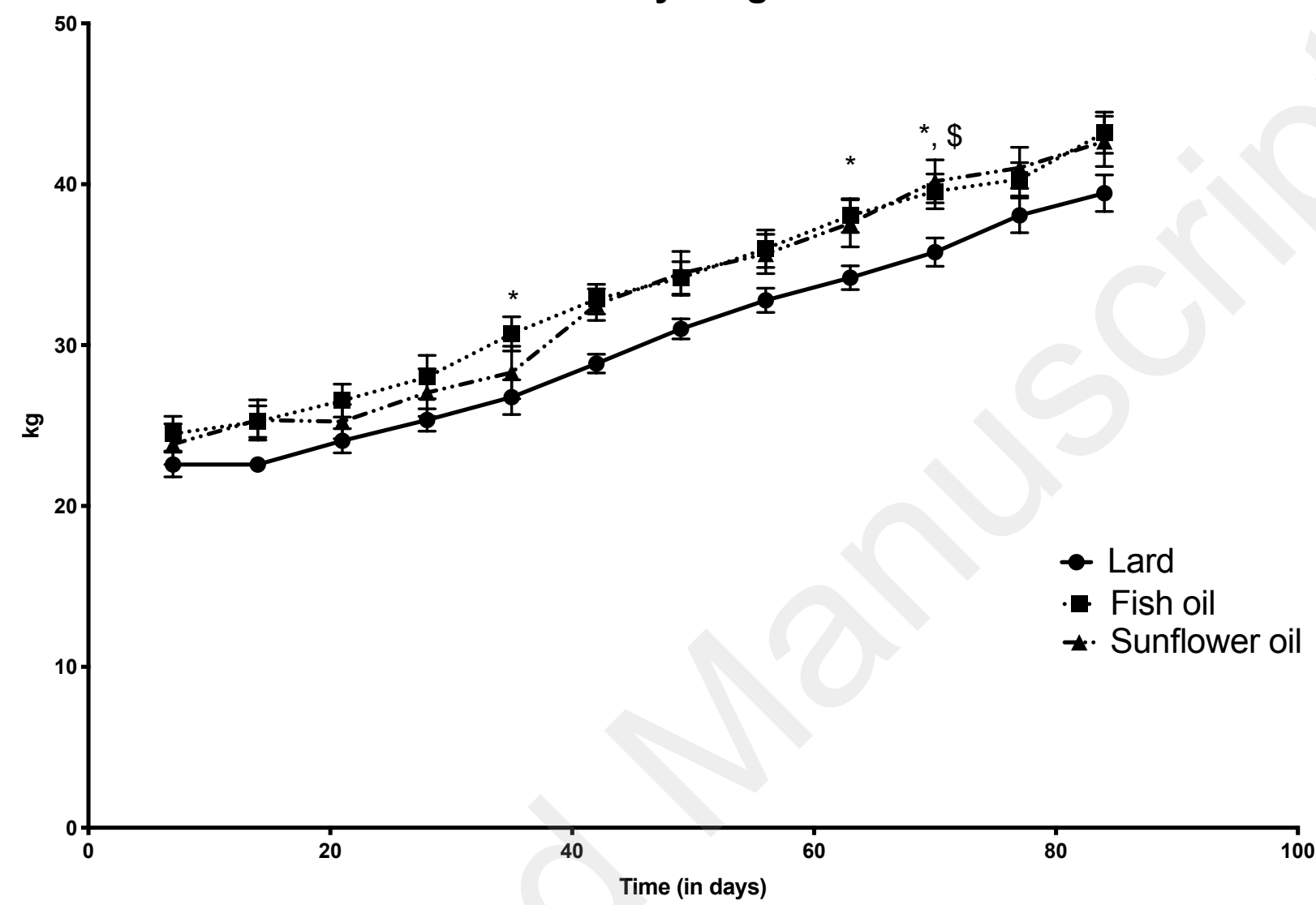

B

Fat Mass

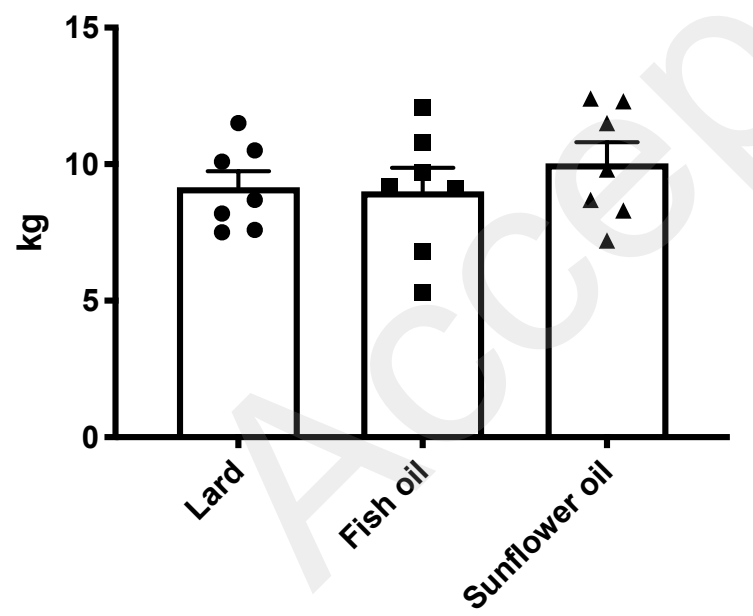

Abdominal fat mass

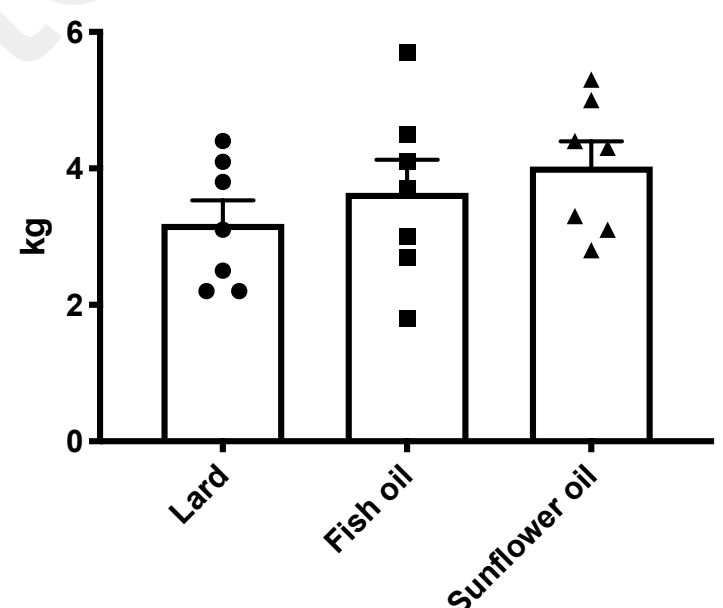

Lean Mass

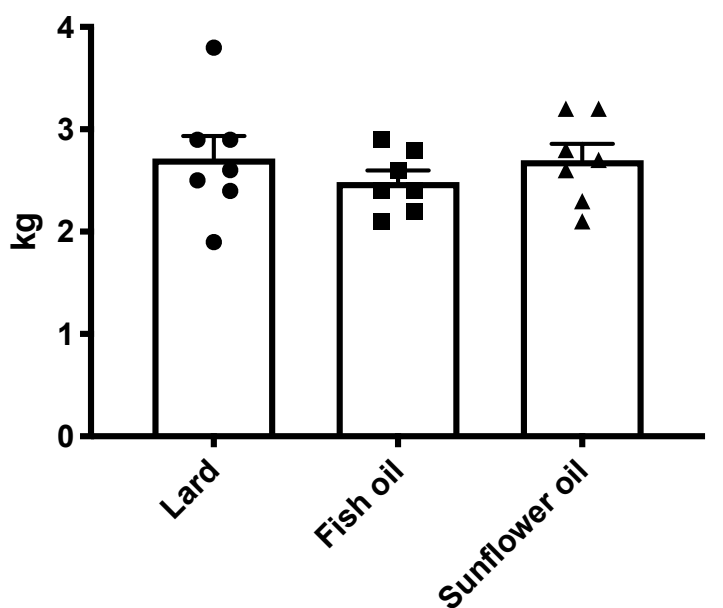


LF Norm

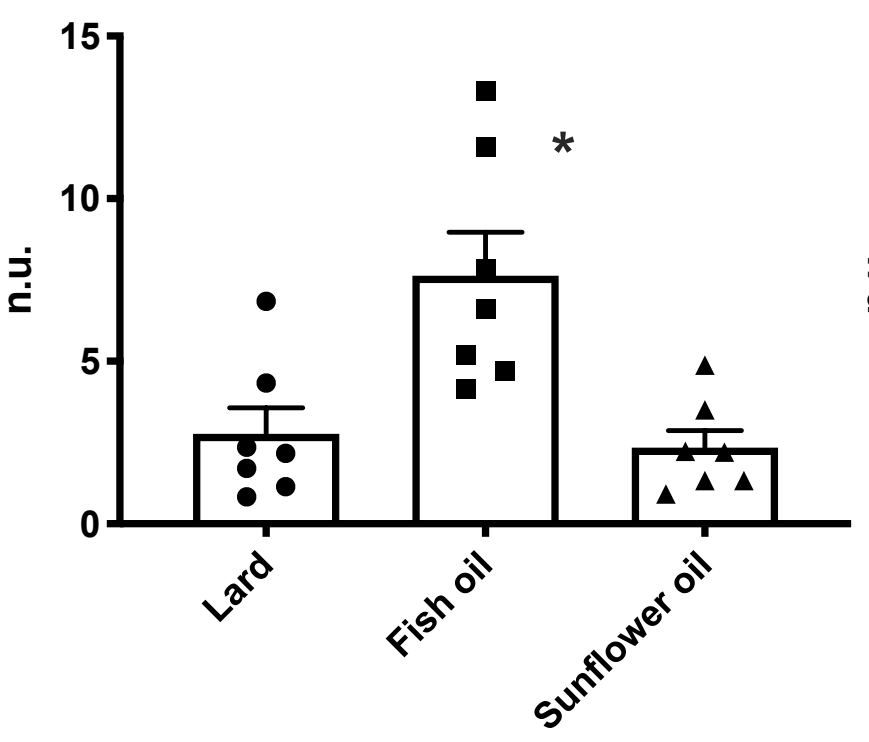

HF Norm

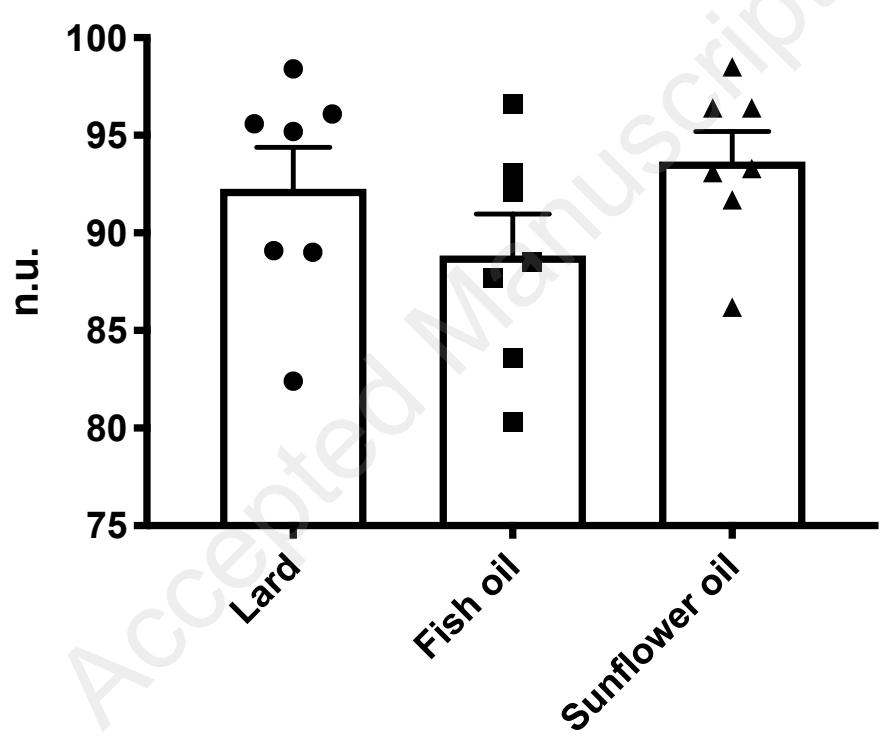

LF/HF

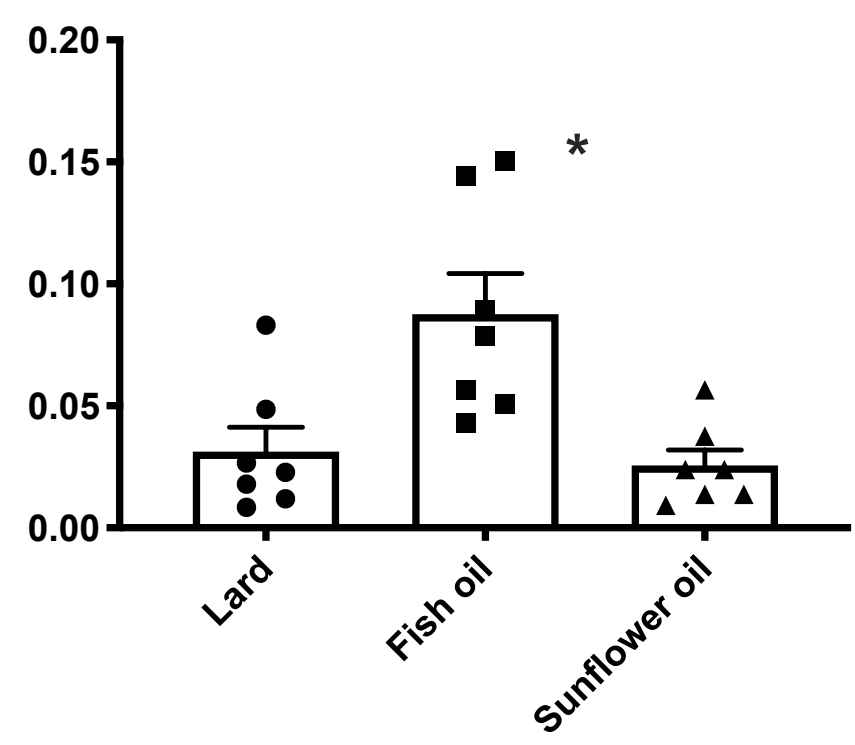




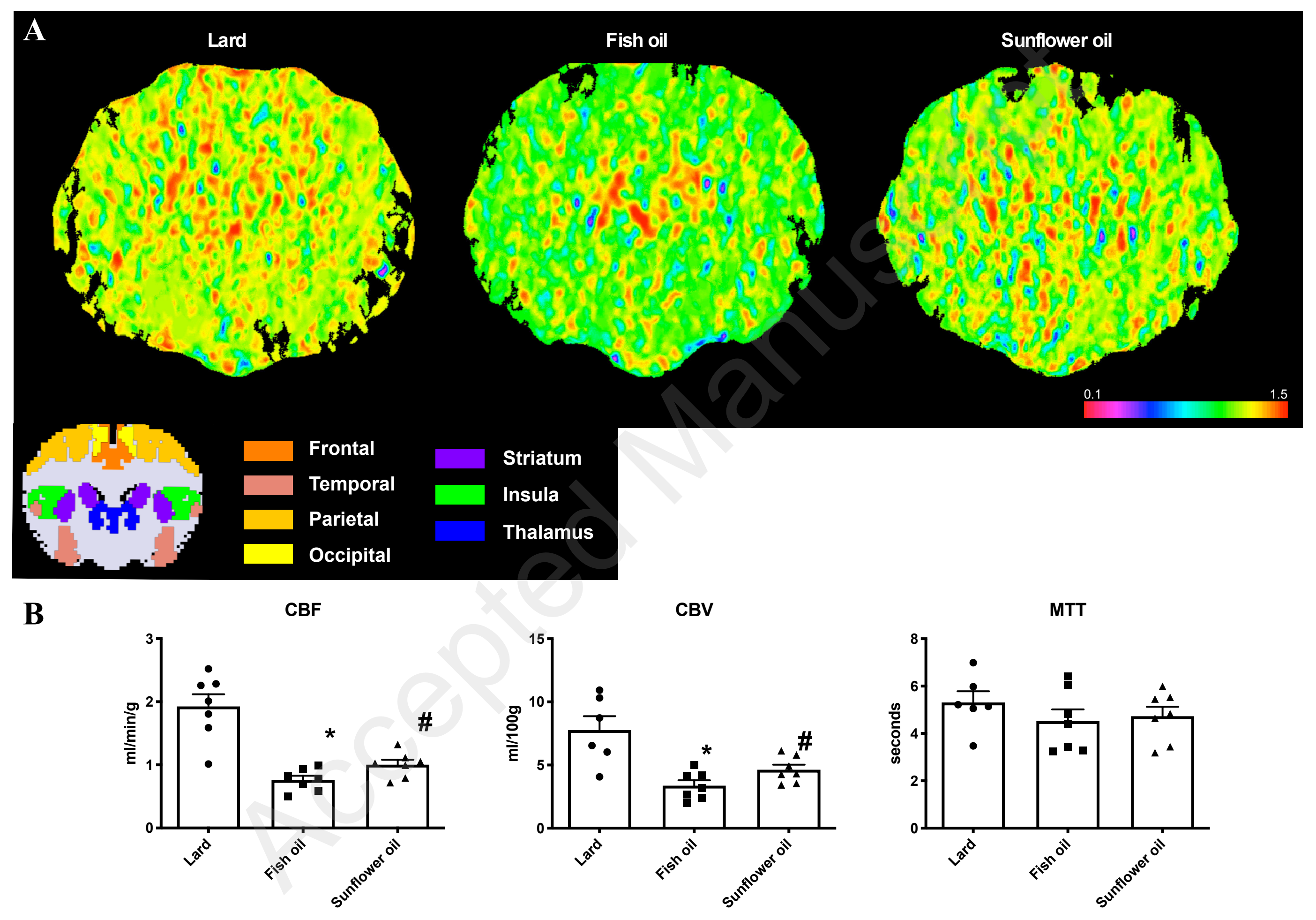




\section{Lard}
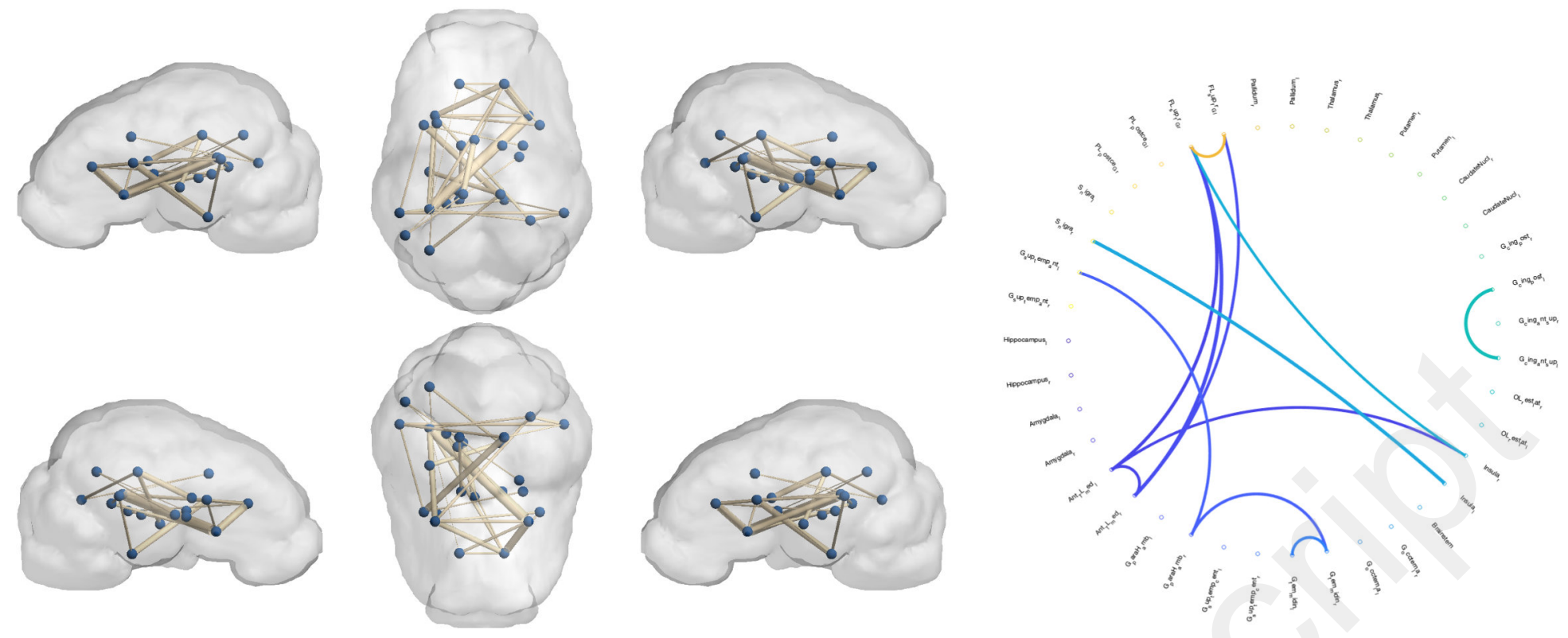

Fish oil
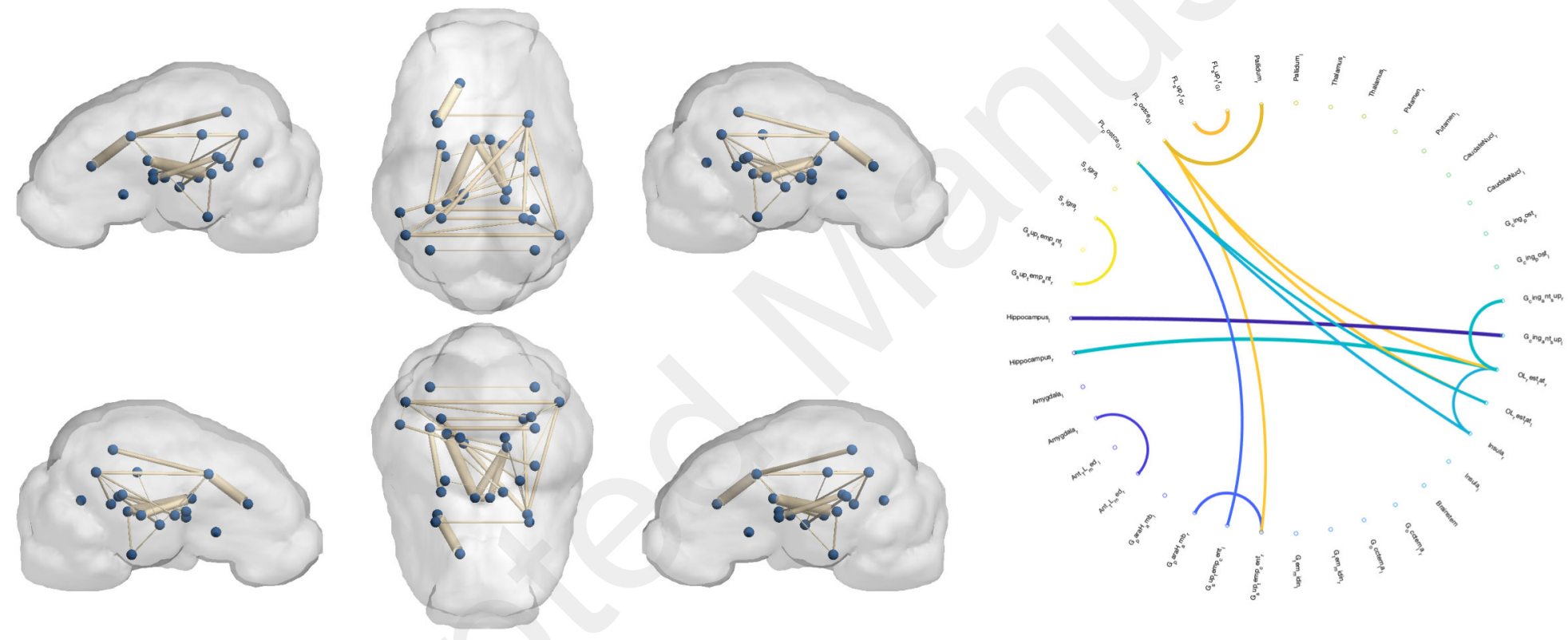

\section{Sunflower oil}
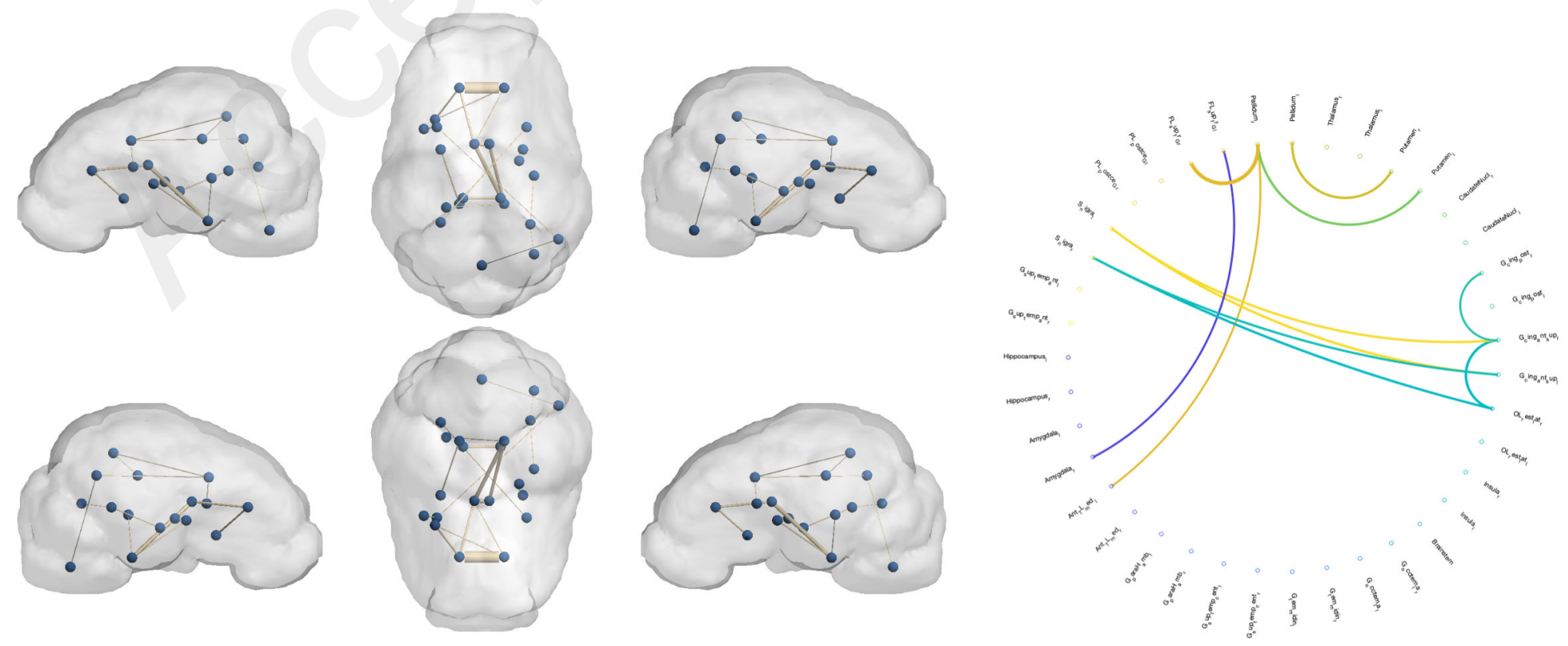